\title{
A polarity comparison timing synchronization estimation for MB-OFDM-based UWB systems
}

\author{
Xue Wang, Zhihong Qian* and Yijun Wang
}

\begin{abstract}
We propose a new approach for timing synchronization estimation with polarity comparison for multi-band orthogonal frequency division multiplexing (MB-OFDM)-based UWB systems. We attempt to locate the start sample of frame sequences by calculating difference of the two cross-correlation functions, between received symbols, the successive received symbols, and predefined preamble sequence. It makes sense to propose polarity comparison and identification ideas to the scenario, the cross-correlation difference exceeding predefined threshold is not unique. If polarities of selected symbols are not all the same, the estimator is put forward to find out a peak of correlation summation to figure out the unique timing point and promote synchronization accuracy. Uniqueness and accuracy of timing synchronization, therefore, could be guaranteed. The performance of the proposed estimator is evaluated by mean square error (MSE) and synchronization probability. The proposed estimator could carry out timing synchronization for MB-OFDM-based UWB systems and make the uniqueness of timing index for sure. The MSEs of the proposed estimator are evidently lower than the reference method for a great deal. Total and exact synchronization probability could get as much as 100 and $96 \%$.
\end{abstract}

Keywords: uniqueness, polarity, timing synchronization, multi-band orthogonal frequency division multiplexing, ultra-wide band

\section{Introduction}

Ultra-wide band (UWB) systems is an attractive technology offering improved ranging precision, high data rate, and enhanced multipath identification. In accordance with terms of FCC [1], UWB is not defined just to pulse transmission [2,3], but can be extended to a continuous transmission technology, as long as absolute signal bandwidth is greater than $500 \mathrm{MHz}$. Multi-band orthogonal frequency division multiplexing (MB-OFDM)-based UWB systems [4], distributing the high-speed data by parallel/serial conversion to a number of sub-channels of relatively low transmission rate, divide the allocated $7.5 \mathrm{GHz}$ spectrum into 14 bands, each with a bandwidth of $528 \mathrm{MHz}$ whereby information is transmitted using OFDM modulation on each band. Its low-power feature offers low interference effects on other wireless technologies working in the frequency range of $3.1-10.6 \mathrm{GHz}$ [5]. The very high data rate (480 Mbps and beyond) capability of UWB technology would provide a compelling

\footnotetext{
* Correspondence: dr.qzh@163.com

College of Communication engineering, Jilin University, $1^{\text {st }}$ building Room 311, Nanhu Avenue No. 5372, Changchun, Jilin province, China
}

cable-replacement wireless technology. OFDM carriers are efficiently generated using a 128-point Inverse Fast Fourier Transform/Fast Fourier Transform (IFFT/FFT). Information is coded across all bands in use to exploit frequency diversity and provide robustness against multipath and interference. MB-OFDM-based UWB system has been proposed for the IEEE 802.15.3a Ultra Wideband standard [6], the new Wireless-USB PHY layer standard, the standard ECMA-368 [7] and ECMA-369.

Synchronization is always a significant issue for any OFDM-based systems. For wireless channel, especially UWB channel, multipath effect is critical, which will cause transmitting signal synchronization loss and subcarrier drifts [8]. There are several frequency offset estimators mentioned in research literatures (e.g., [9-16]). Meanwhile, the exact start position is ought to be confirmed to demodulate received data correctly, for timing error could cause inter carrier interferences (ICI) and inter symbol interferences (ISI), which will lead to orthogonality loss of OFDM subcarriers and degrade system performance.

The physical layer scheme of ECMA-368 adopts preamble-based mode, therefore, synchronization in 
MB-OFDM-based UWB systems is data-aided. Schmidl and Cox [17] defined a preamble structure with two identical parts. Timing synchronization is implemented by finding out the peak of pilot correlation, which introduces the timing synchronization idea that bases on preamble design. Studies [18-20] are on the basis of Schmidl's approach. The algorithms they proposed could realize timing synchronization, but are restricted by multipath effect. The above researches have solved timing issue for a great deal. Since the preamble structure for MB-OFDM-based UWB systems has been defined in literature [6] already, corresponding schemes are in need to improve system synchronization capability. Adaptive timing synchronization estimators are proposed in [21,22], which are implemented by using energy ratio of received symbols. Peak detection [23], based on literature [17], and maximum likelihood estimator [24], based on literature [15], are applied to the systems. Some researchers consider implementing timing synchronization for MB-OFDM-based UWB systems by a defined operation (e.g., energy difference operation [25], or correlation difference operation [26]) to work out threshold contrast. Those types of estimators mitigate the multipath effect restrict, and make synchronization with an acceptable synchronization probability, but have some serious threshold limitation. The first sample that exceeds the threshold may not be the right timing sample. What's worse, the threshold set for the current SNR environment may not suit for other SNRs. Therefore, the threshold setting plays an important role in the performance of timing synchronization for the systems. Besides, if the samples exceeding the threshold are all taken into account, the consequence of running the algorithms would conclude more than one timing index when the threshold is a small one. Otherwise, none of timing index would be obtained when the threshold is relatively big. They both do not make sense for timing synchronization. The characters of correlation are also widely used in the design of timing synchronization estimators $[27,28]$. Concerning the work of timing synchronization of MB-OFDM-based UWB systems, a majority of estimators proposed are of great performance for TFC1 (or equivalently 2), but the performances for TFC3 (or equivalently 4) are ignored, which should be taken into consideration, for they are also widely used.

In this article, we analyze classical preamble-based timing synchronization estimators and a typical estimator for MB-OFDM-based UWB systems. We propose a timing synchronization estimator with three steps. Polarity identification and summation peak operation are carried out to guarantee the uniqueness of timing synchronization and promote timing synchronization probability for both TFC1 (or TFC2) and TFC3 (or TFC4). Our proposed estimator offers significant mean square error
(MSE) improvement over the reference estimator. Meanwhile, the synchronization of our estimator could achieve a total synchronization probability as much as $100 \%$ and an exact synchronization probability of $96 \%$ in CM1 and TFC1. In CM2 and TFC3, the proposed estimator could get a total synchronization probability of $100 \%$ and an exact probability of $93 \%$.

The key contributions of this article include

- A new timing scheme utilizing polarity features of preambles. The approach is proposed totally according to the definition in protocol ECMA-368. It develops the unique characteristic of MB-OFDM-based UWB systems.

- Performance improvement to thresholds-based synchronization estimations. The proposed approach could combine with thresholds-based synchronization schemes to improve their performances in low SNR environments. Simulation results indicate that more than $90 \%$ synchronization probability improvement could be achieved when the proposed scheme is appended to correlation-based symbol timing synchronization (CBTS).

- Uniqueness guarantee of timing synchronization. The resolution could absolutely get a unique timing simple, which is really meaningful for MB-OFDM-based UWB systems timing.

- Less restriction in threshold selection comparing to traditional thresholds-based timing synchronization schemes. The process of polarity comparing could improve systems timing property as well as guarantee unique timing sample in an extend threshold range. Therefore, we do not need to choose a severe threshold value, but in a certain range.

- More flexible for various time frequency modes. Traditional thresholds-based approaches define an operation to get a timing sample, which does not change in different time frequency modes. The proposed approach utilizes polarity features. If a different time frequency mode is adopted, the polarities distributions change adaptively. So, the proposed scheme is more flexible for different time frequency modes.

The rest of the article is organized as follows: Section 2 presents the MB-OFDM system specifications, characters of UWB channel with channel measurement parameters, MB-OFDM signal model, and the analysis of the outstanding estimators in literatures. The proposed timing synchronization estimator is described in Section 3. Section 4 shows the simulation results and discussions. Conclusion and summary are provided in Section 5.

\section{System description}

\subsection{MB-OFDM specifications}

In MB-OFDM-based UWB systems [6], the carrier frequency hops with a predefined set of carrier frequencies according to a time frequency code (TFC). ECMA 
standard specifies seven types of TFCs, which are defined in [6]. Preamble patterns are associated with TFCs. Each preamble pattern is constructed by 24 synchronization sequences and 6 channel estimation sequences. Figure 1 indicates the structure of preamble.

The 24 synchronization sequences are constructed by 21 packet synchronization sequences (PS) and 3 frame synchronization sequences (FS). Timing synchronization for MB-OFDM-based UWB systems is to find the exact start of FS from the received symbols, so as to receive and demodulate the received symbols correctly. For preambles 1 and 2, which are associated with TFC1 and TFC2, the first 21 sequences of synchronization sequences are PS, and the other three are FS. For preamble patterns 3 and 4, which are defined according to TFC3 and TFC4, the combination of PS and FS are interleaved. For all the preamble patterns, the polarities of PS and FS are all opposite. Polarities for PS are positive, whereas for FS are negative. The features could be used for timing synchronization.

\subsection{Signal model}

In MB-OFDM-based UWB systems, zero-padded (ZP) prefix is used instead of the conventional cyclic prefix. Symbols are constructed by suffixing $32 \mathrm{ZP}\left(N_{\text {pre }}\right)$ and 5 guard $\left(N_{\mathrm{g}}\right)$ samples to $128(N)$ length IFFT sequence. The total number of samples in one OFDM symbol is $N_{\text {total }}=N+N_{0}, N_{0}=N_{\text {pre }}+N_{\mathrm{g}}$.

Suppose frequency offset has been estimated and compensated perfectly, transmitted sequence in band ' $b$ ' can be expressed as

$$
S_{b}=\left\{s_{b}(0), s_{b}(1), \cdots, s_{b}(n-1), s_{b}(n), s_{b}(n+1), \cdots\right\} ; n \geq 0 .
$$

The received sequence considering channel response is addressed as

$$
R_{b}=\left\{r_{b}(0), r_{b}(1), \cdots, r_{b}(n-1), r_{b}(n), r_{b}(n+1), \cdots\right\} ; n \geq 0 .
$$

The $n$th sample of $l$ th OFDM transmitted symbol in band ' $b$ ' is

$$
r_{b}(l, n)=\sum_{i=0}^{L_{b}-1} s_{b}(l, n-i) h_{b}(i)+w_{b}(l, n) ; 1 \leq l \leq L,
$$

where $s_{b}(l, n)$ is the $n$th sample of $l$ th symbol in band ' $b$ ', $w_{b}(n, l)$ is the corresponding AWGN sample, $h_{b}(i)$ is the IEEE 802.15.3a UWB RF channel impulse response [29] in band ' $b$ '. The general equation is described as

$$
h(t)=X \sum_{l=0}^{L} \sum_{k=0}^{K} \alpha_{k, l} \delta\left(t-T_{l}-\tau_{k, l}\right),
$$

where $\alpha_{k, l}$ is channel coefficient for $k$ th ray of $l$ th cluster, $T_{l}$ is the delay of $l$ th cluster, $\tau_{k, l}$ is the delay of $k$ th ray related to $l$ th cluster arrival time, $X$ is the lognormal shadowing on the amplitude. More details are provided in [29].

\subsection{Timing synchronization}

Timing synchronization is to find the exact start of FS in MB-OFDM-based UWB systems, so as to demodulate the received symbol accurately. Before we proceed, let us briefly analyze the classical timing synchronization schemes presented in $[17,18]$, and a typical estimator for MB-OFDM-based UWB systems in [26].

\subsubsection{Analysis of Schmidl's and Minn's approaches}

ECMA-368 has already defined the preamble structure of MB-OFDM UWB. The defined preamble is started with 21 consecutive sequences (PS), which fulfills the conditions of Schmidl's [17] and Minn's [18] approaches. We try to utilize Schmidl's and Minn's ideas to implement timing synchronization in MB-

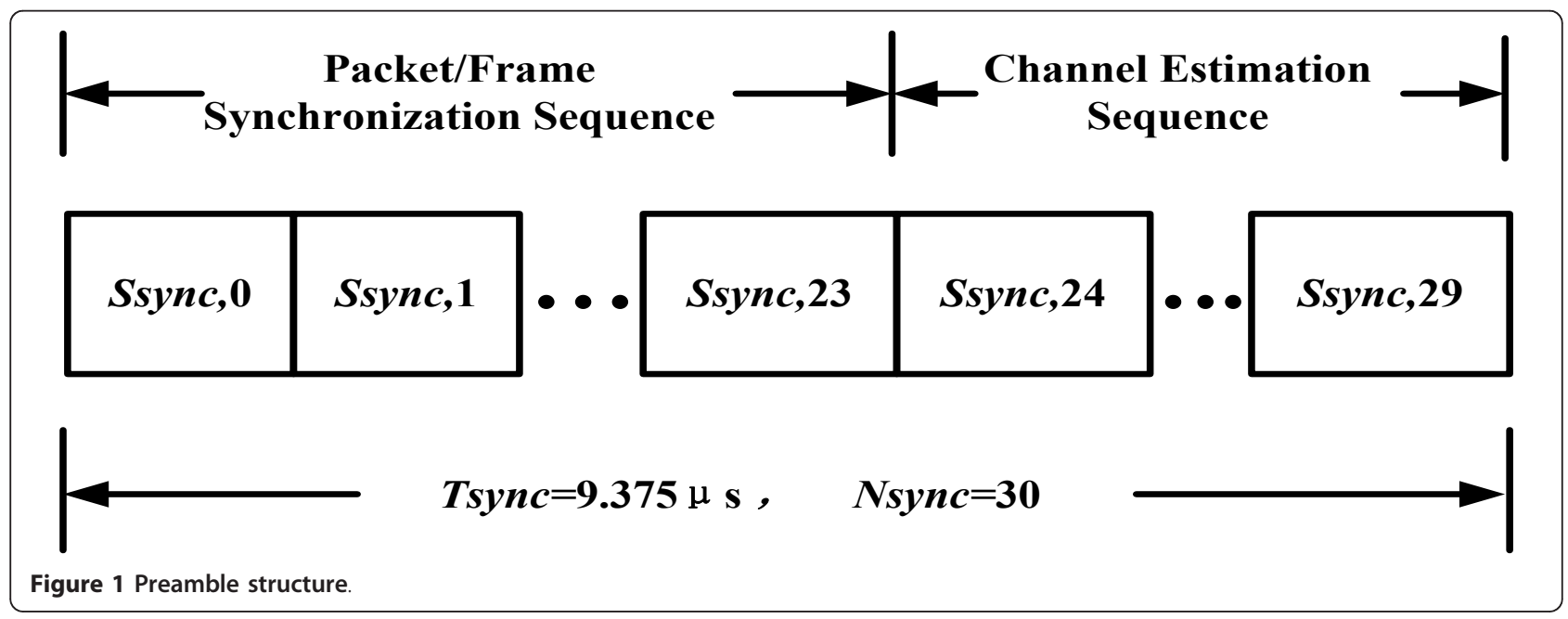


OFDM-based UWB systems. The results of timing matrixes for preambles 1 (or equivalently 2 ) and 3 (or equivalently 4) are given in Figures 2 and 3, respectively.

We can see that the Schmidl's method achieves an evidently maximum value at the start sample of both PS sequences and FS sequences. However, it is not unique maximum value, which means that it will select more than one timing index if used in timing synchronization. The Minn's method could get a maximum value at the first sample of PS, but not FS. Therefore, both Schmidl's and Minn's methods could not find the right timing index under the preamble structure in MB-OFDMbased UWB systems directly.

\subsubsection{CBTS method}

Sen et al. [26] proposed a CBTS method, which could obtain the right timing index by operations on the difference of two consecutive received symbols. The estimator can be expressed as follows.

The cross correlation between the $l$ th received OFDM symbol and preamble sequence is addressed as

$$
R_{b}(l, \tau)=\sum_{n=0}^{N-1} r_{b}(l, n+\tau) d^{*}(n) ; \quad 0 \leq \tau \leq N_{\text {total }}-1, \quad 1 \leq l \leq L
$$

where $\{d\}=\left\{d_{1}, d_{2}, \ldots . . ., d_{N}\right\}$ is the predefined preamble sequence. The cross correlation between the $(l+1)$ th received sample and preamble sequence is

$$
R_{b}(l+1, \tau)=\sum_{n=0}^{N-1} r_{b}(l+1, n+\tau) d^{*}(n) ; \quad 0 \leq \tau \leq N_{\text {total }}-1, \quad 1 \leq l \leq L-1
$$

The correlation difference of adjacent correlations is addressed as

$$
D_{b, \tau}=R_{b}(l+1, \tau)-R_{b}(l, \tau), \quad 1 \leq l \leq L-1 .
$$

If $R_{b}(l, \tau)$ and $R_{b}(l+1, \tau)$ belong to the same pilot period, the difference $D_{b, \tau}$ will be amplitude of noise in theory, which is expected to be of a small value. On the conditions that $R_{b}(l, \tau)$ and $R_{b}(l+1, \tau)$ belong to different pilot periods, which means that $R_{b}(l, \tau)$ belongs to PS while $R_{b}(l+1, \tau)$ belongs to FS, the difference $D_{b, \tau}$ may get to a significant value. By setting a threshold $\lambda$, timing point can be achieved.

We simulate the difference of correlations between the received symbols for both preamble patterns 1 and 3 associated with TFC1 and TFC3, respectively, the results of which are given in Figures 4 and 5. They show the outcomes of correlation difference in four conditions: (A) CM1 with SNR $=10 \mathrm{~dB}$; (B) CM1 noiseless environment; (C) CM2 SNR = $10 \mathrm{~dB}$; (D) CM2 noiseless environment.

From the simulation we can see that the channel models are of randomness and multipath effect, so that the difference result corresponding to the right timing index is not always the maximum one. What's worse, when a threshold is selected, quantity of difference result exceeding threshold may be more than one or none on the contrary. For example, if we set the threshold as 60, the algorithm could obtain more than one timing samples in the situation of Figure 5A, C, D.

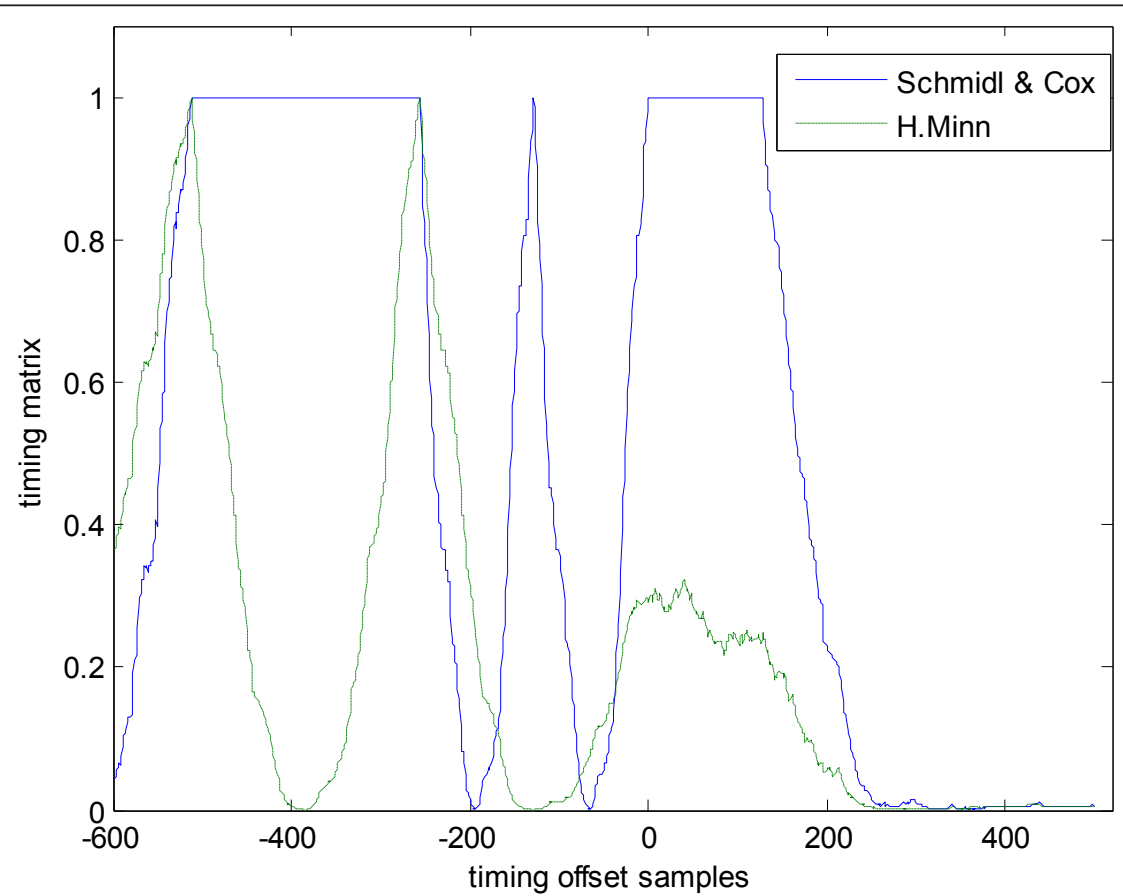

Figure 2 Timing matrixes for preamble 1 (or equivalently 2) in MB-OFDM-based UWB systems 


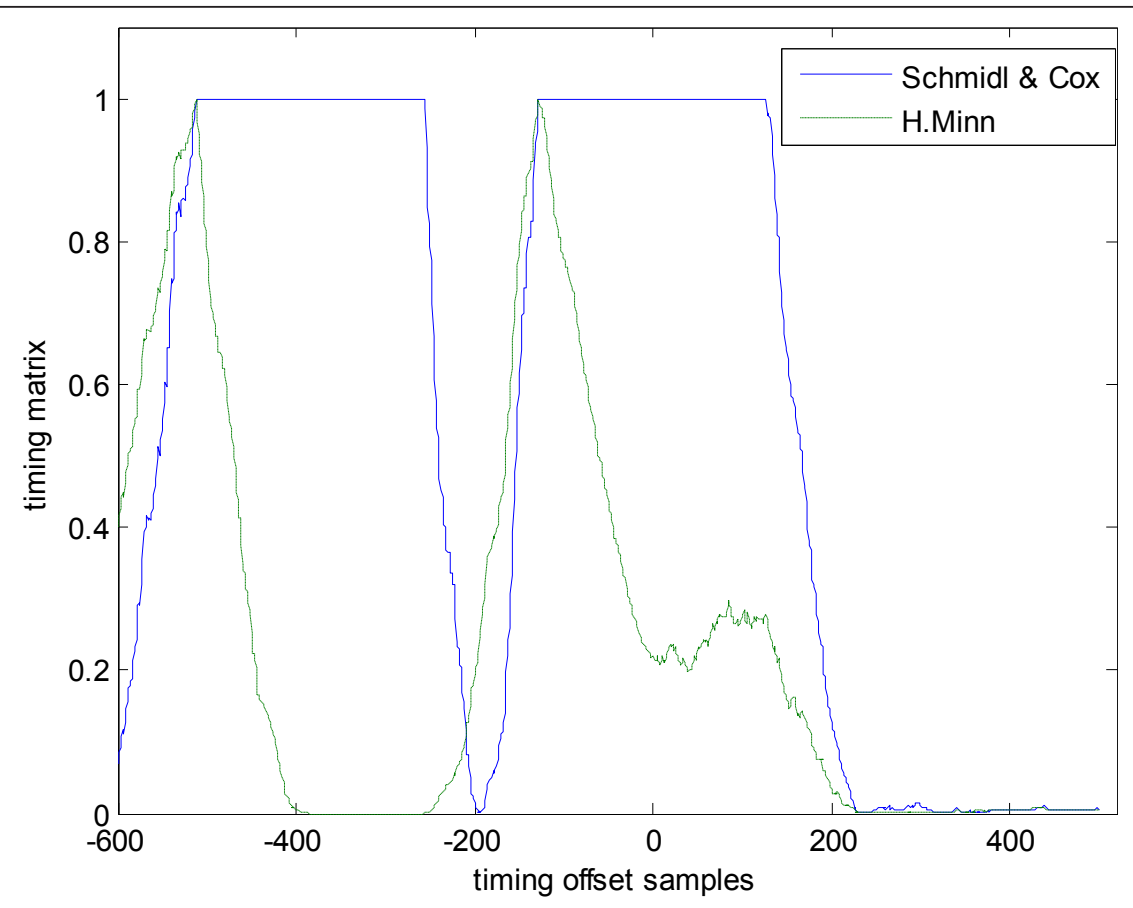

Figure 3 Timing matrixes for preamble 3 (or equivalently 4) in MB-OFDM-based UWB systems
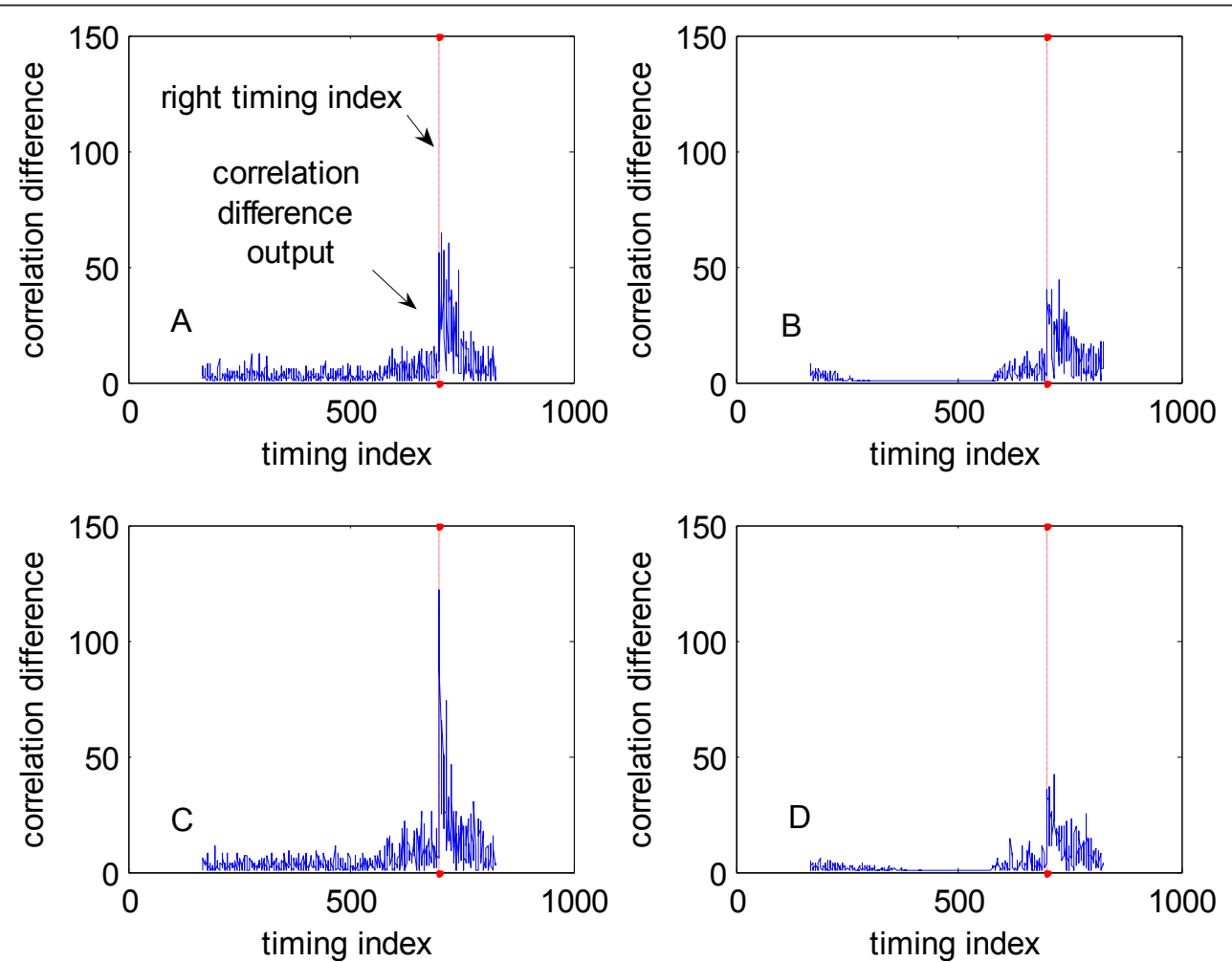

Figure 4 Correlation difference output for preamble 1 (or equivalently 2). (A) $C M 1$, SNR $=10 \mathrm{~dB}$. (B) $C M 1$ noiseless. (C) $C M 2, S N R=10 \mathrm{~dB}$. (D). CM2, noiseless. The received timing index varies from 0 to 1000. The real blue lines in figures are the correlation difference operation results of adjacent received symbols, and the dotted red lines show the exact timing index. The meanings of different lines of Figure 5 are the same as this figure. 

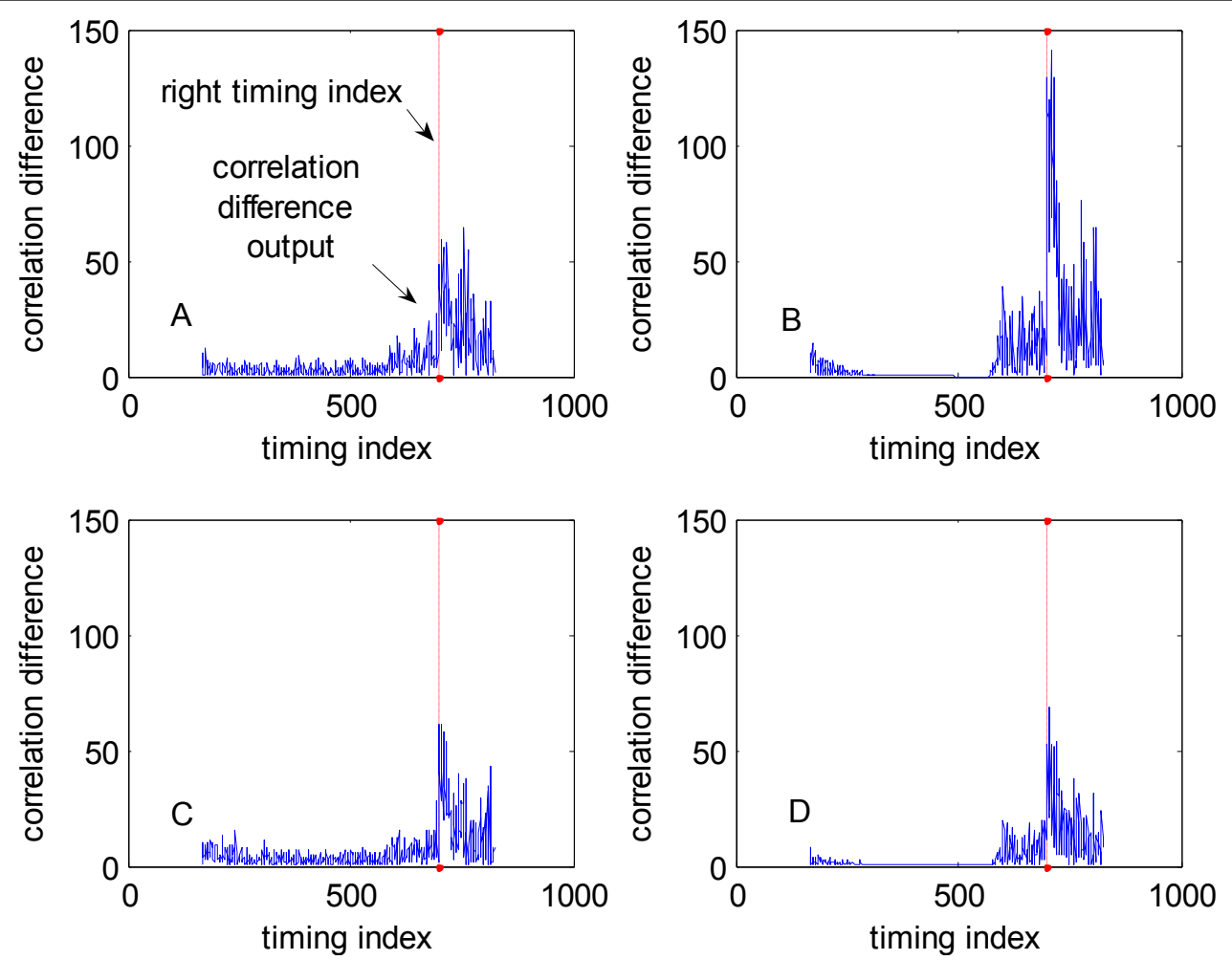

Figure 5 Correlation difference output for preamble 3 (or equivalently 4). (A). CM1, SNR = 10 dB. (B) CM1 noiseless. (C). CM2, SNR = 10 dB. (D). CM2, noiseless. The received timing index varies from 0 to 1000 .

When we set a larger threshold, maybe the difference results are out of range, and no sample could be found out. Therefore, threshold is a key point for timing. And threshold should change as the channel environment changing. Meanwhile, we should notice that the features of correlation difference for preamble pattern 3 (or equivalently 4) are not distinct.

\section{The proposed timing synchronization estimator}

As analysis we have done above, classical preamblebased timing synchronization estimators are not suit for UWB systems very well. Among the methods specially proposed for MB-OFDM-based UWB systems, the CBTS method is of remarkable property, which is a typical threshold-based estimator. However, CBTS is of threshold restriction, and does not match other preamble patterns well. Besides, the uniqueness of correlation difference is uncertainty. If the uniqueness of timing synchronization could not be guaranteed, it will lead to inefficiency of timing algorithms. Aiming to solve the problem, we address a novel timing estimator with three steps, which could decrease the impact of threshold in a great deal, promote the flexibility for both preambles 1 (or 2) and 3 (or 4), enhance the stability of estimators, as well as the uniqueness of timing sample.

\subsection{First step: cross-correlation difference}

Calculating difference of the two cross-correlation functions, one, between a received symbol and predefined preamble sequence, the other, between the successive received symbol and predefined preamble sequence, and then, calculating the difference of next two cross-correlation functions, where the "successive received symbol" mentioned above acts as the first received symbol, one after another in this way, which is addressed in (7). Then, we set a threshold $\lambda$, and the timing index (indexes) corresponding to the received symbols exceeding $\lambda$ is (are) estimated to be timing result for the first step.

$$
\begin{aligned}
& \operatorname{abs}\left(D_{b, \tau}\right)>\lambda \\
& \tau_{\text {first }}=(l+1) * N_{\text {total }}+\tau
\end{aligned}
$$

where $\tau_{\text {first }}$ is the timing index (indexes) achieved in the first step by correlation difference.

\subsection{Second step: polarity comparison and identification} Define $M$ as the total number of the timing indexes achieved in the first step, $M \in\left[1, N_{\text {total }}\right]$. If $M=0$, the threshold should be reconsidered, being a bit less perhaps, for there is no sample exceeding the current 
threshold. Otherwise, $\tau_{\text {first, } m}$ is the $m$ th timing index achieved in the first step, $1 \leq m \leq M$. $\tau_{\text {final }}$ is the final timing index. The implementation process of our timing estimator is different with $M$ values. The value of $M$ can be classified as following two scenarios.

(1) $\boldsymbol{M}=\mathbf{1}$

The very one is the final timing index

$$
\tau_{\text {final }}=\tau_{\text {first }, 1}
$$

(2) $M>1$

The samples obtained in the first step should be selected for one or two more rounds. To decrease algorithm complexity and promote algorithm accuracy, we attempt to compare the polarities of the symbols $r_{b}\left(\tau_{\text {first }}\right.$, m) corresponding to $\tau_{\text {first, } m}$.

Define

$$
P J\left(\tau_{\text {first }, m}\right)=p\left(r\left(\tau_{\text {first }, m}\right)\right) \oplus p(d)
$$

where $p\left(r\left(\tau_{\text {first }, m}\right)\right)$ presents the preamble polarity of the $\tau_{\text {first, } m}$ th received symbol, and $p(d)$ is the polarity of predefined preamble sequence. $P J\left(\tau_{\text {first, } m}\right)$ is XOR operation of received symbols and predefined sequence.

If $P J\left(\tau_{\text {first, }} m\right)$ equals to 0 , the $\tau_{\text {first, } m}$ th received symbol and predefined sequence are with the same polarity, which means the $\tau_{\text {first, } m}$ th received symbol belongs to PS; otherwise, they are of different polarities, and the $\tau_{\text {first, } m}$ th received symbol belongs to FS.

Sum up $P J\left(\tau_{\text {first }} m\right)$ for $m$ ranging from 1 to $M$, the results of which can be classified as

$$
P J_{\text {sum }}=\sum_{m=1}^{M} P J\left(\tau_{\text {first }, m}\right)=\left\{\begin{array}{l}
M \\
(0, M) . \\
0
\end{array}\right.
$$

If polarities of all the selected timing indexes match polarity of predefined sequences, $P J_{\text {sum }}$ would equals to 0 , which indicates $\tau_{\text {first, } m}$ belongs to PS. Therefore, the bigger $\tau_{\text {first, } m}$ is, the closer $r_{b}\left(\tau_{\text {first, } m}\right)$ is, to FS. Then the right start of FS can be estimated as

$$
\tau_{\text {final }}=\max \left(\tau_{\text {first }, m}\right)+1
$$

If all the polarities of selected timing indexes are opposite with the predefined sequence, $P J_{\text {sum }}$ would equals to $M$, which denotes $\tau_{\text {first, } m}$ belongs to FS. Thus, the smaller $\tau_{\text {first, } m}$ is, the closer $r_{b}\left(\tau_{\text {first, } m}\right)$ is, to the start of FS. Then the right start of FS can be estimated to be

$$
\tau_{\text {final }}=\min \left(\tau_{\text {first }, m}\right)
$$

On the case that timing indexes selected in the first step consist of not only indexes corresponding to symbols belonging to PS, but also those corresponding to symbols belonging to $\mathrm{FS}, P J_{\text {sum }}$ would be neither 0 nor
$M$. We need one more step to choose the right synchronization result guaranteeing uniqueness of timing synchronization and timing accuracy.

\subsection{Third step: uniqueness guaranteeing}

Define

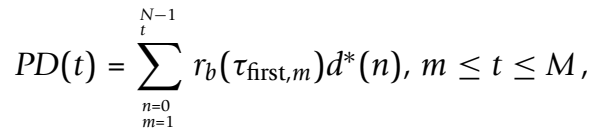

Considering the preamble polarity, all the PS polarities are positive, whereas all the FS polarities are negative. With received symbols increasing, $P D(t)$ increases. When FFT window is aligned with FS, $P D(t)$ decreases because of the opposite polarities. Therefore, the time index corresponding to the maximum $P D(t)$ is expected to be the final timing index.

$\tau_{\text {final }}=\tau_{\text {first, } \operatorname{argmax}(\operatorname{abs}(P D(t)))}$

where $\tau_{\text {final }} \in \tau_{\text {first }}$, and $\tau_{\text {final }}$ is expected to be unique.

\section{Simulation and discussion}

We now present the simulation results for IEEE 802.15.3a [5] channel models 1 and 2 [29]. The channel is time invariant for duration of the preamble. And in simulations we have done, the MB-OFDM-based UWB systems with $N=128, N_{\text {pre }}=32, N_{\mathrm{g}}=5$, and carrier frequencies $f=$ 4.125 MHz is set according to the specifications in [6]. We have illustrated in the previous sections that there are 24 synchronization sequences in one MB-OFDM-based UWB frame, 21 PS sequences and 3 FS sequences. In the process, we set $L=6$ to run simulations and analyze the algorithm for convenience, which is constructed by PS and FS sequences as [PS1, PS2, PS3, PS4, FS1, FS2] for preamble pattern 1 (or 2) and [PS1, PS2, PS3, FS1, PS4, FS2] for preamble pattern 3 (or 4). We adopt TFC1 and TFC3 to simulate the estimator in preambles 1 and 3, respectively. All the simulation results are achieved over 1000 times estimations.

The classical timing synchronization algorithms, such as Schmidl's [17] and Minn's methods [18], are not suitable for the synchronization of the MB-OFDM-based UWB systems, which are demonstrated by Figures 2 and 3 . Therefore, we will compare our synchronization procedure with CBTS [26], which is of a significant synchronization ratio among synchronization algorithms.

\subsection{Timing results quantity}

To demonstrate the effectiveness of the proposed estimator, we calculate the number of synchronization samples obtained by simulation for the proposed estimator and the correlation difference. The averaged number in noiseless environments of CM1 and CM2 are given in 
Table 1 The number of timing samples in CM1

\begin{tabular}{lllll}
\hline Threshold $(\boldsymbol{\lambda})$ & \multicolumn{4}{l}{ The number of timing synchronization samples in CM1 } \\
\cline { 2 - 5 } & \multicolumn{1}{l}{ TFC1 } & TFC3 & The proposed \\
\cline { 2 - 5 } & Correlation difference & The proposed & Correlation difference & 1 \\
\hline 30 & 21.70 & 1 & 24.54 & 1 \\
40 & 13.69 & 0.98 & 15.40 & 0.97 \\
50 & 8.59 & 0.92 & 8.72 & 0.90 \\
60 & 5.45 & 0.83 & 5.46 & 0.85 \\
70 & 4.08 & 0.73 & 3.52 & 0.78 \\
80 & 1.88 & 0.61 & 2.39 & 0.53 \\
90 & 0.98 & & 0.99 & \\
\hline
\end{tabular}

Tables 1 and 2, respectively. The thresholds are set in the range ' $\lambda$ in $[30,90]$ '.

Comparisons between correlation difference and the algorithms we proposed demonstrate that the phenomenon of multi-timing synchronization samples is critical. The number of timing symbols achieved after synchronization of correlation difference is more than 1 , when threshold ranges from 30 to 80 . The proposed estimator could guarantee the uniqueness of synchronization results. From the results shown in tables above, when threshold is set larger than 50, average number of our proposed estimator is less than one. Nevertheless, average number of correlation difference is considerably over one. The phenomenon indicates that if the threshold is set over some value, correlation difference would get more than one synchronization sample, or on the contrast, get none, both scenarios would lead to the inefficiency of timing synchronization. The proposed algorithm could guarantee uniqueness of timing synchronization, if threshold is not chosen excessively big. That feature is of great significance for system synchronization.

\subsection{MSE performance}

To explore the threshold restriction of proposed estimator and existing estimators, simulations with different threshold are carried out. We define the simulation of 100 times as one group, and we could get an MSE value of the timing results after one group simulation. To fully consider the MSE performance, we run the simulation for 10 groups, which means totally 1000 times simulation. Then we can get 10 MSEs for a threshold. We select the optimal MSEs and average MSE values of all the simulation groups to analyze features of estimators. Under noiseless environments of CM1 and CM2, taking TFC1 and TFC3, setting the threshold ranging from 30 to 90 , we work out the MSE performance of the proposed estimator comparing with the CBTS [26], as presented in Figures 6 and 7, respectively.

We can see from the MSE curves in Figures 6 and 7 that the proposed timing synchronization estimator has a much smaller MSE in general. In the simulation environment of CM1 and TFC1, the proposed estimator could get an optimal MSE as small as 0.09 and a least average MSE of 0.35667. The optimal MSE and average MSE of CBTS are 0.09 and 0.96202, respectively. The optimal MSE of the proposed method in low threshold are evidently lower than CBTS and threshold based method. Taking the MSEs achieved if threshold is 30 as an example, the optimal MSE of the proposed estimator is 0.44 , while that of CBTS is 3.68. The average MSE of the proposed is 5.026, and that for CBTS is 79.522, which is about 15 times of the proposed one. In the situation of larger threshold, the proposed estimator and CBTS are nearly the same, for the number of samples derived after the correlation difference are limited,

Table 2 The number of timing samples in CM2

\begin{tabular}{lllll}
\hline Threshold $(\boldsymbol{\lambda})$ & \multicolumn{4}{l}{ The number of timing synchronization samples in CM2 } \\
\cline { 2 - 5 } & \multicolumn{1}{l}{ TFC1 } & TFC3 & The proposed \\
\cline { 2 - 5 } & Correlation difference & The proposed & Correlation difference & 1 \\
\hline 30 & 21.54 & 1 & 25.94 & 1 \\
40 & 14.70 & 1 & 13.30 & 0.97 \\
50 & 5.79 & 0.98 & 9.36 & 0.93 \\
60 & 4.61 & 0.95 & 4.57 & 0.79 \\
70 & 2.86 & 0.86 & 2.58 & 0.71 \\
80 & 1.73 & 0.72 & 2.02 & 0.59 \\
90 & 0.94 & 0.41 & 1.01 & \\
\hline
\end{tabular}




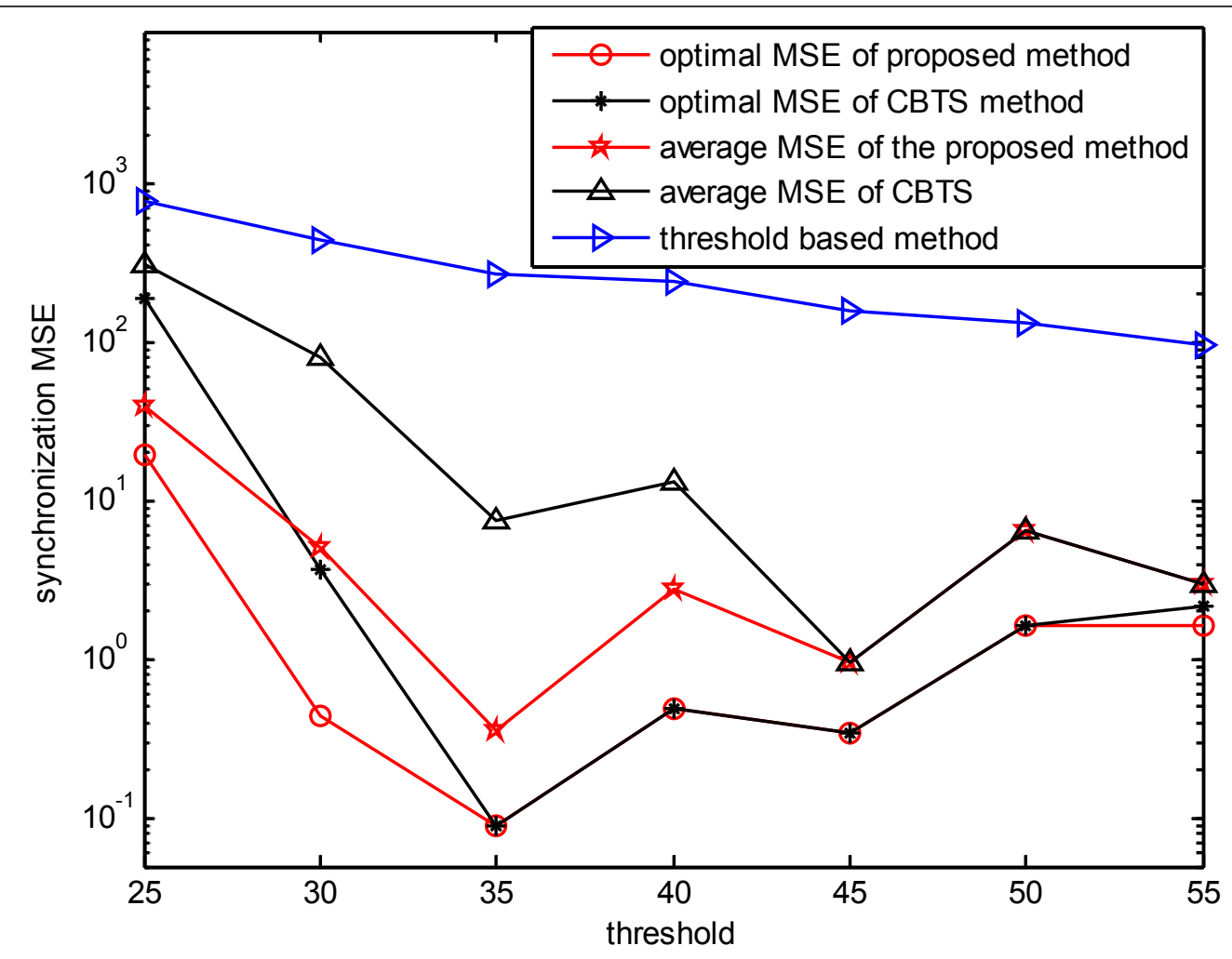

Figure 6 MSE performance with different thresholds in CM1 and TFC1.

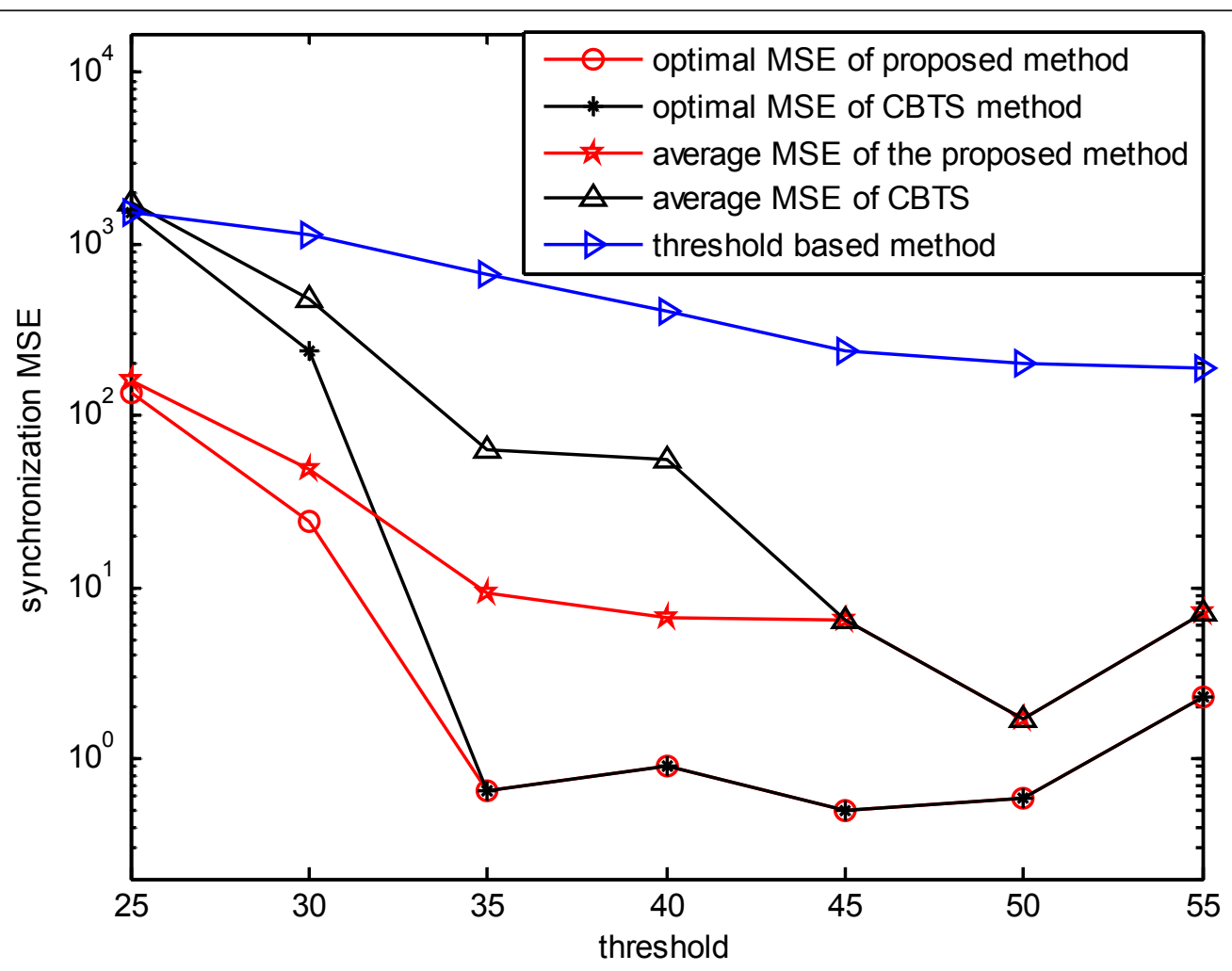

Figure 7 MSE performance with different thresholds in CM1 and TFC3. 
sometimes even without any sample. Therefore, these two algorithms have to choose an optimal result from the limited samples. Average MSEs of the proposed method are better than those of CBTS, especially in small threshold condition, which indicates that the proposed estimator has a better stability. For TFC3, the proposed estimator also performs better than CBTS in general. The average MSEs of the proposed estimator demonstrate its better stability. The proposed estimator decreases the limitation of threshold as well.

Figures 8 and 9 give optimal MSEs and average MSE performance of the three kinds of estimators in CM2.

The optimal MSEs of the proposed estimator and CBTS in CM2 and TFC1 are nearly the same with a smallest optimal MSE of 0.08. Average MSE of the proposed estimator is 0.298, which is smaller than 1.01391, the smallest average MSE of CBTS, For TFC3, the optimal MSE and average MSE of the proposed estimator are better than those of CBTS for threshold under 45 . According to average MSEs of the two estimators, the proposed algorithm provides excellent stability.

We notice that both the proposed estimator and CBTS perform well in particular if threshold is set in the range ' $\lambda$ in $[30,90]$ '. When threshold is larger than 50 , the uniqueness of timing synchronization cannot be guaranteed. Therefore, we set threshold as 35 , and SNR varies from $0 \mathrm{~dB}$ to $30 \mathrm{~dB}$ to see the performance of estimators. Figures 10,11, 12, and 13 present the MSE in $\mathrm{CM} 1$ \& $\mathrm{TFC} 1, \mathrm{CM} 1$ \& $\mathrm{TFC} 3, \mathrm{CM} 2$ \& $\mathrm{TFC} 1$, and CM2 \& TFC3, with different SNRs, respectively.

Simulation results indicate that the CBTS performs well in CM1 and TFC1. In other conditions, the MSE performances of the proposed estimator are all better than CBTS. What's more, the averages MSE of the proposed one in the four conditions are all lower than that of CBTS for at least $16 \mathrm{~dB}$, which means that the proposed estimator provides a much more significant stability than CBTS.

For threshold-based estimator, which refers to the correlation difference, when threshold goes larger, the MSE gets smaller. That is because if we choose a larger threshold, fewer timing samples will be achieved after correlation difference. Therefore, the samples that are more close to the right timing sample are chosen. Figures 4 and 5 demonstrate that the overall timing operation goes in some kind of rules, but the specific value is with some degree of random, which means that correlation difference will get a relatively large value that is not always over the threshold. Therefore, the existing and uniqueness of timing cannot be guaranteed. No matter with different thresholds, or in different SNR environments, it is always with a big MSE. Thus, it would not

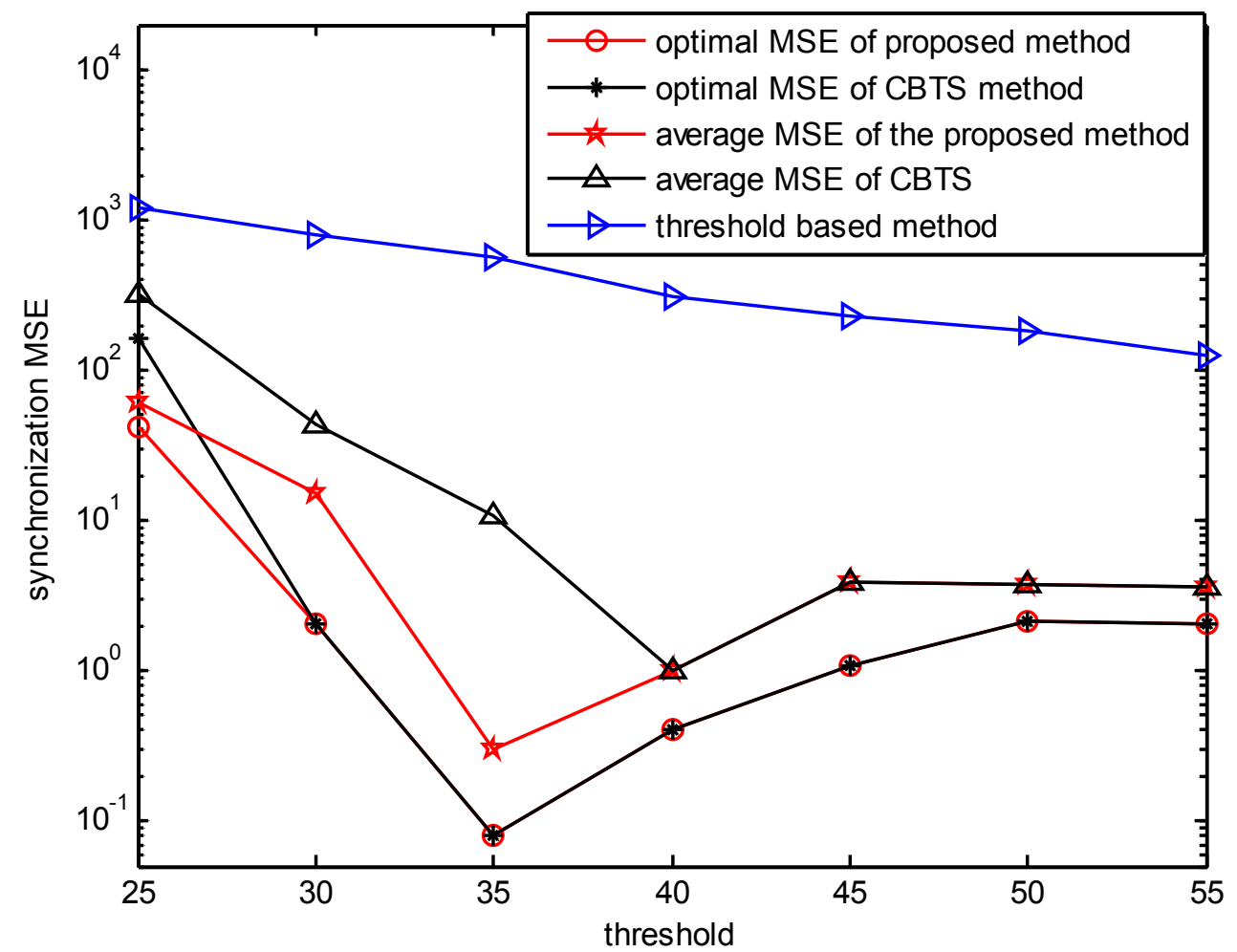

Figure 8 MSE performance with different thresholds in CM2 and TFC1. 


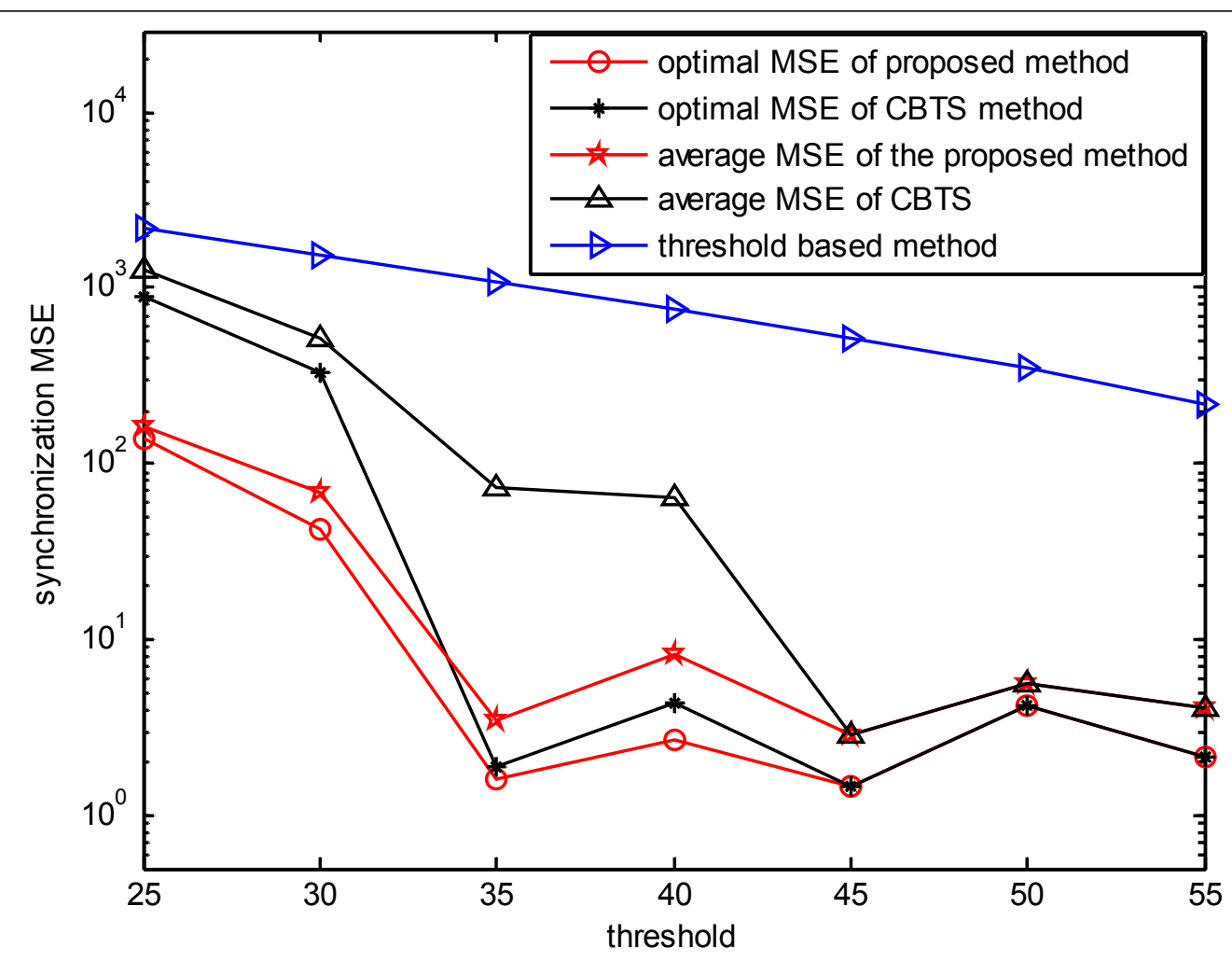

Figure 9 MSE performance with different thresholds in CM2 and TFC3

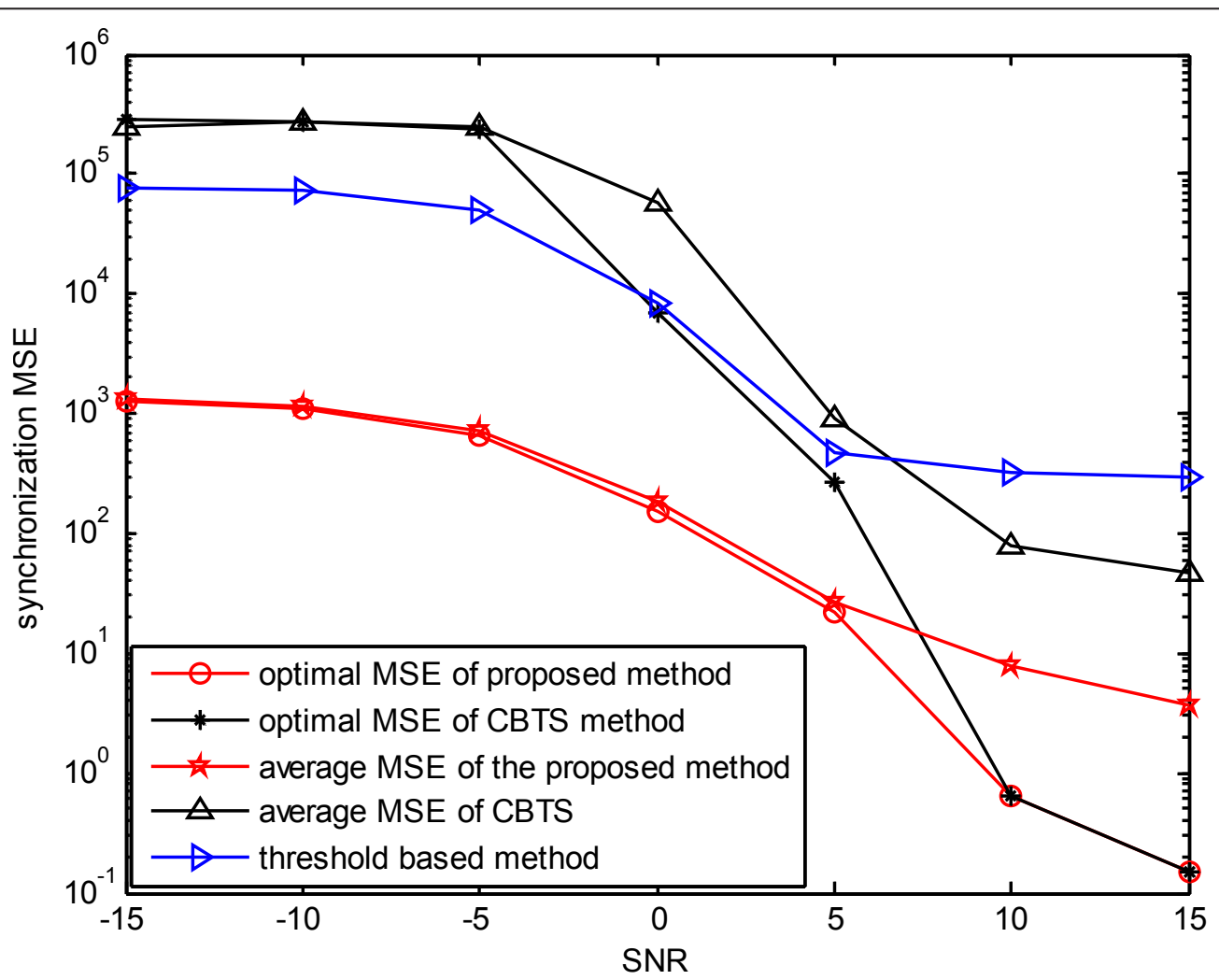

Figure 10 MSE performance with different SNR in CM1 and TFC1. 


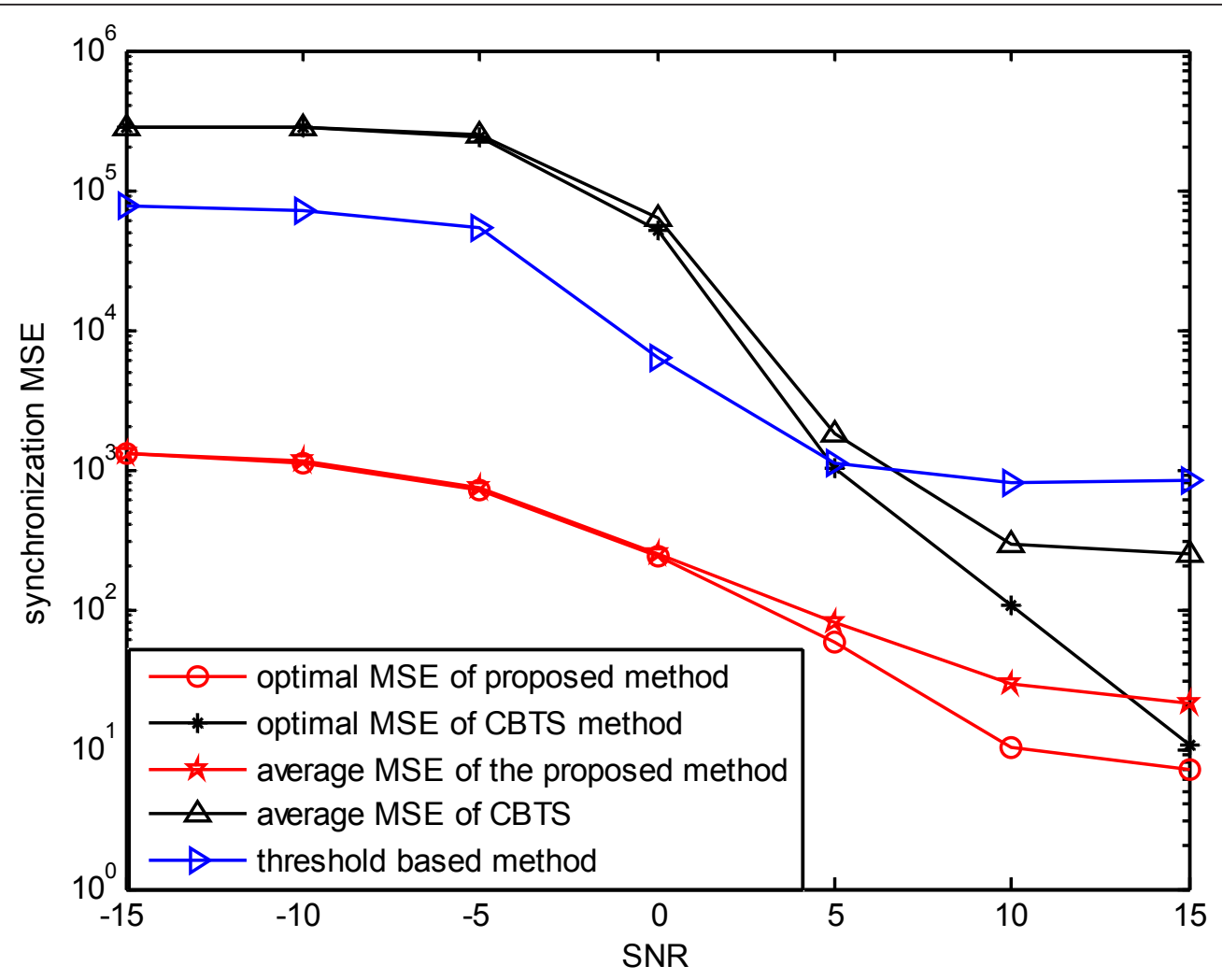

Figure 11 MSE performance with different SNR in CM1 and TFC3.

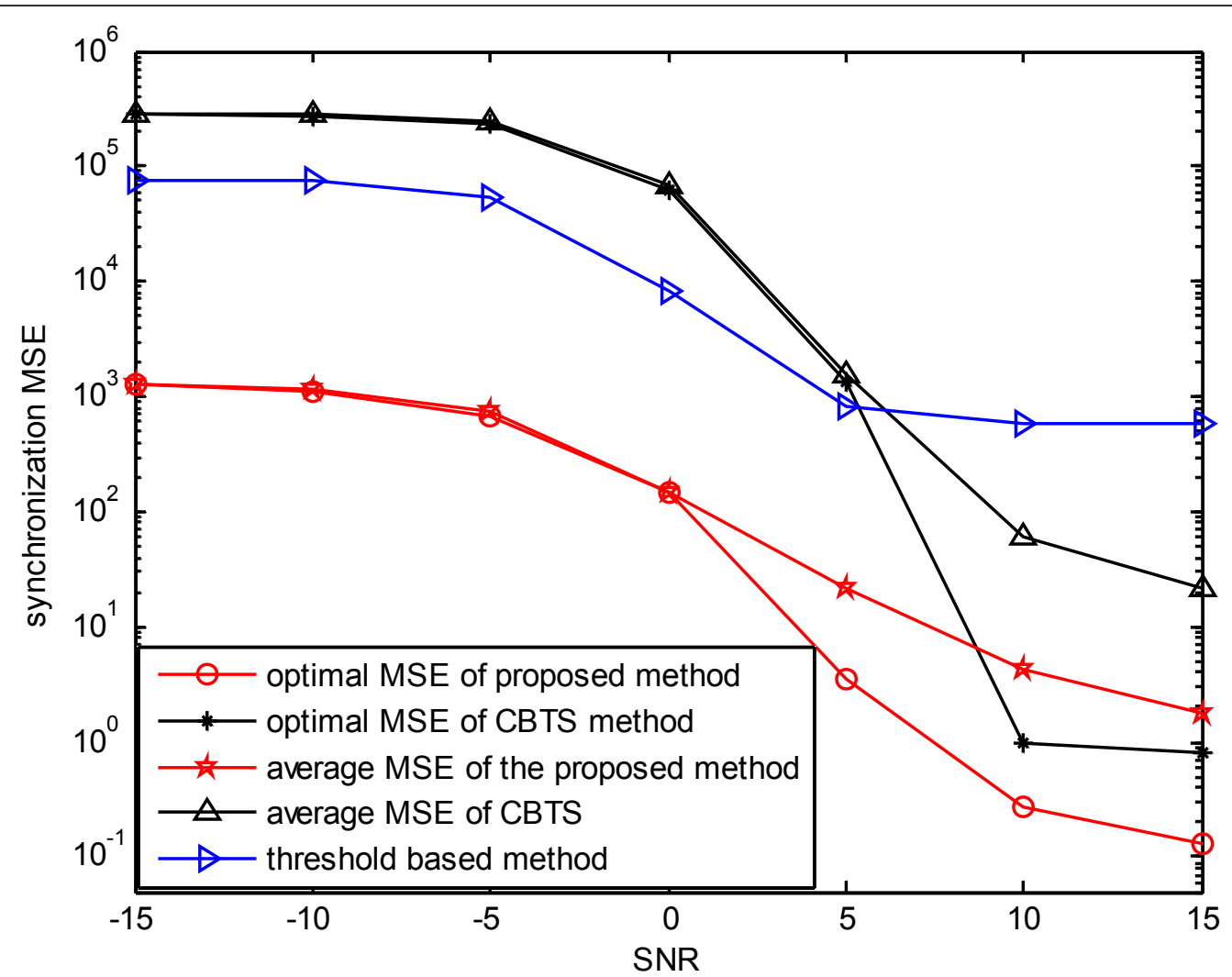

Figure 12 MSE performance with different SNR in CM2 and TFC1 


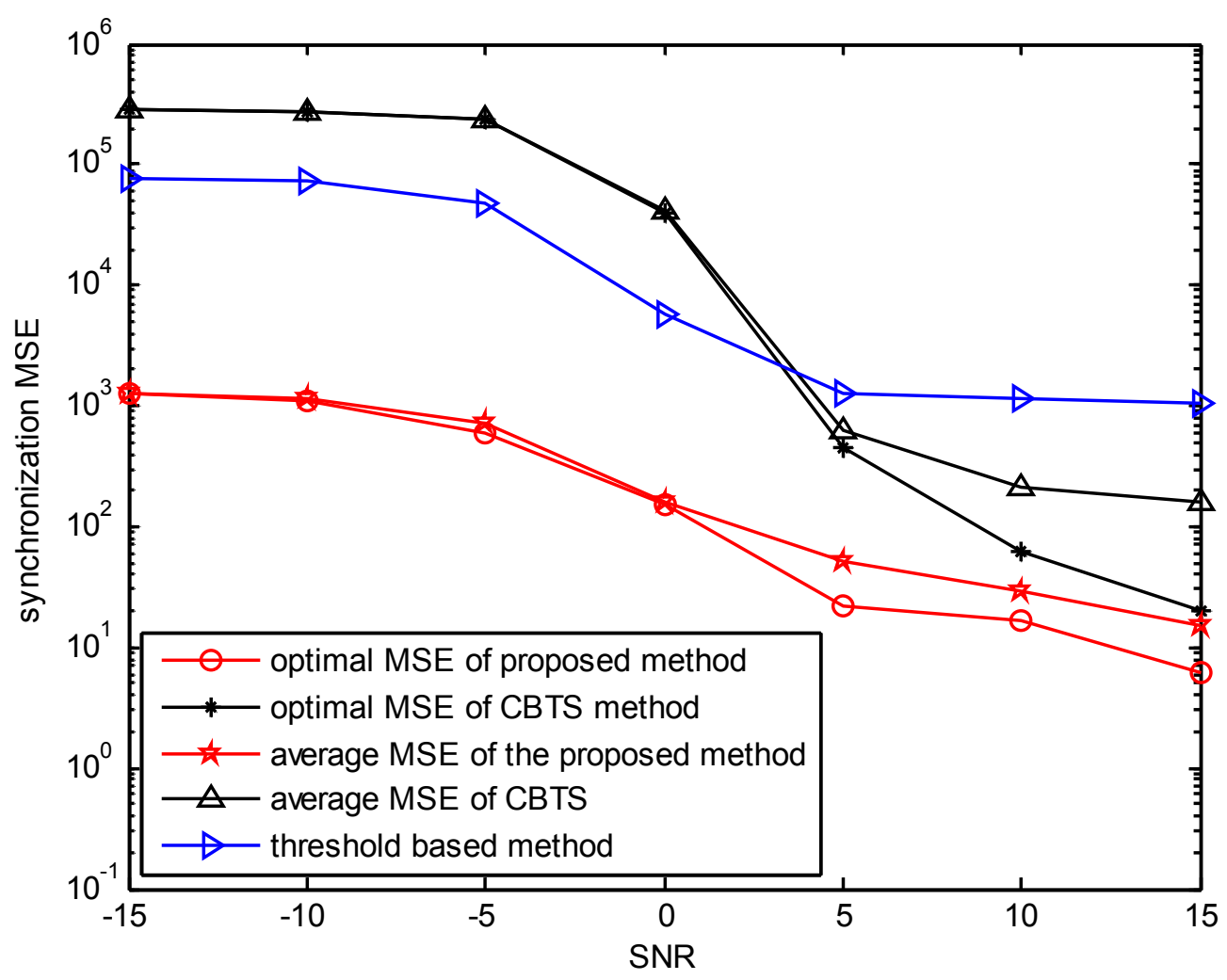

Figure 13 MSE performance with different SNR in CM2 and TFC3.

work if we want to get a timing index just by catching samples exceeding a predefined threshold.

\subsection{Synchronization probability}

In order to analyze performance of estimators more comprehensively, we evaluate the synchronization probability of our proposed estimator and the CBTS [26], in CM1 and CM2, taking TFC1 and TFC3, respectively. The total probability $P_{\text {total }}$ is defined as follows.

$$
P_{\text {total }}=P_{\text {exact }}+P_{\text {zp }}
$$

where $P_{\text {exact }}$ is the probability that the estimation sample is exactly the right start point of FS. $P_{\mathrm{zp}}$ is the probability that the estimation sample locates in the range of $\mathrm{ZP}$, which would not introduce inter symbol interface.

Figures 14 and 15 give the synchronization probability with different thresholds in CM1, CM2, TFC1, and TFC3. The channel environment is noiseless, which is convenient for us to get the optimal synchronization capacity.

The maximum total and exact synchronization probabilities of the proposed estimator among the results we achieved are 99 and $96 \%$ for TFC1 in the channel environment of CM1. These of CBTS are both $96 \%$. For TFC3, the maximum and exact probabilities of the proposed estimator among the results we achieved are 98 and $92 \%$. These of CBTS are 96 and $91 \%$. So, the synchronization capacity of CBTS is lower than that of the proposed algorithm for both TFC1 and TFC3. The improvement is evident in particular if threshold is relatively small. The reason why synchronization probabilities of the two estimators in larger thresholds are not as much as those in smaller thresholds is that the samples exceeding the threshold of correlation difference are limited. Then the right timing sample would probably be missed by this operation. So, the two estimators have to select timing index from the elected samples. They could only choose a sample mostly close to the start of FS, which finally leads to the outcome of MSE and synchronization probabilities.

The maximum total and exact synchronization probabilities of the proposed estimator among the results we achieved are 97 and $95 \%$ for TFC1 in CM2. And for CBTS, they are 96 and 95\%. For TFC3, the maximum and exact probabilities of the proposed estimator among the results we achieved are 98 and $93 \%$. These of CBTS are 95 and $92 \%$. The synchronization probability is not as large as that for TFC1. It is due to the intermittent of FS in preamble pattern 3 (or equivalently 4). The preamble pattern 3 (or 4) is not constructed by a 

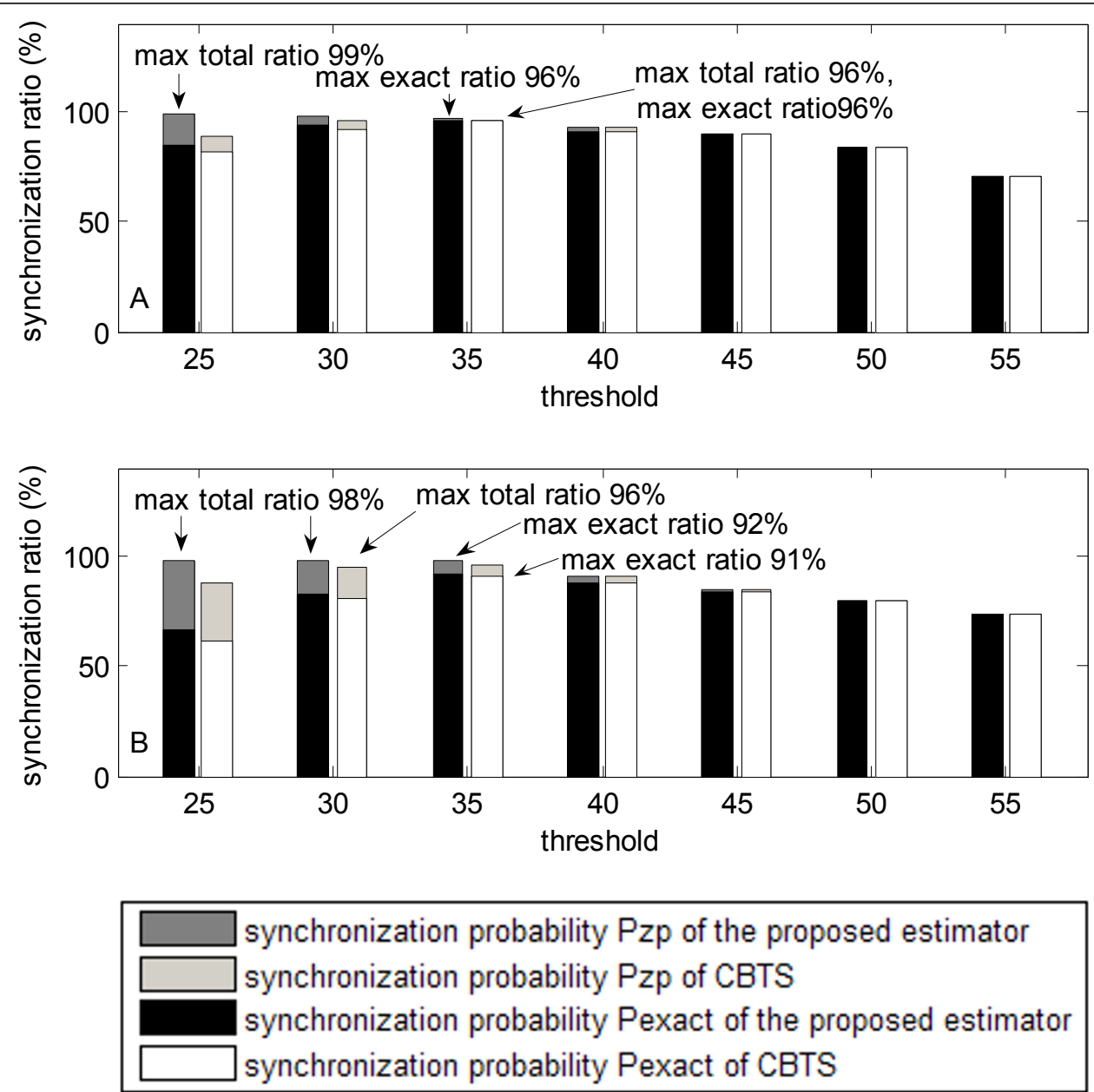

Figure 14 Synchronization probabily with different thresholds in CM1: (A) Preamble 1. (B) Preamble 3. Dark gray bars are the synchronization probabilities $P_{z p}$ of the proposed estimator; light gray bars are the synchronization probabilities $P_{z p}$ of CBTS; black bars are the synchronization probabilities $P_{\text {exact }}$ of the proposed estimator; white bars are the synchronization probabilities $P_{\text {exact }}$ of CBTS. The meanings of different color bars in latter figures are the same with those in this figure.

consecutive PS sequences or FS sequences, and the pattern is a cross combination of PS and FS. Therefore, the cross-correlation features are not as good as that of preamble 1 (or equivalently 2). Our proposed estimator does not only utilize the features of correlation difference, but also the polarities of different symbols. Thus, the performance of the proposed one is also better than CBTS that only considers features of correlation difference. Totally speaking, the proposed estimator could get a more outstanding synchronization probability and stability than the CBTS for both preamble patterns 1 and 3 in CM1 and CM2. If threshold is 35, the two estimators could get an optimal exact synchronization probability.

Figures 16 and 17 give the synchronization probabilities in different SNR environments.

The charts indicate that the total synchronization probabilities of the proposed estimators all over 90\%, which demonstrates the stability of the proposed estimator once more. The merits of our proposed approach are in evidence especially in environment of $\mathrm{SNR}<0$ $\mathrm{dB}$. Both the proposed scheme and literature estimation could get scarcely any exact synchronization probabilities. Total synchronization probabilities of CBTS are rather low, which are $0 \%$ when SNR $=10 \mathrm{~dB}$. But, the proposed one could get almost $99 \%$, even or $100 \%$ total synchronization probabilities among the results we achieved. We notice that the maximum syncrhonization probabilities in Figures 14 and 15 do not reach $100 \%$ as in Figures 16 and 17. The reasons why they are different are simulation randomness. Therefore, the results indicate the maximum probabilities which are achieved among the simulations we have done, which would be a larger one if we proceed more times of simulations. As we have illustrated before, estimated timing point 

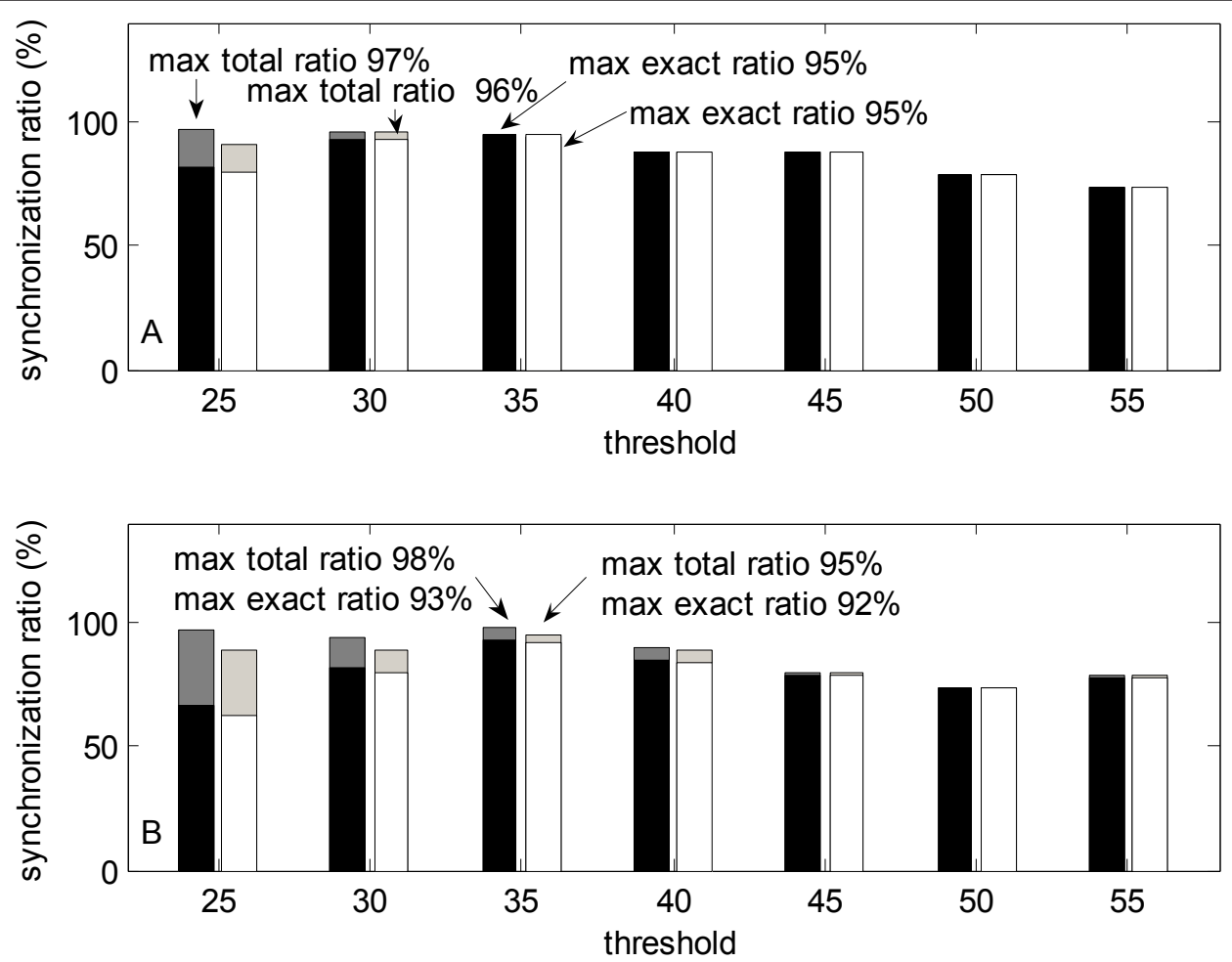

Figure 15 Synchronization probability with different thresholds in CM2: (A) Preamble 1. (B) Preamble 3.

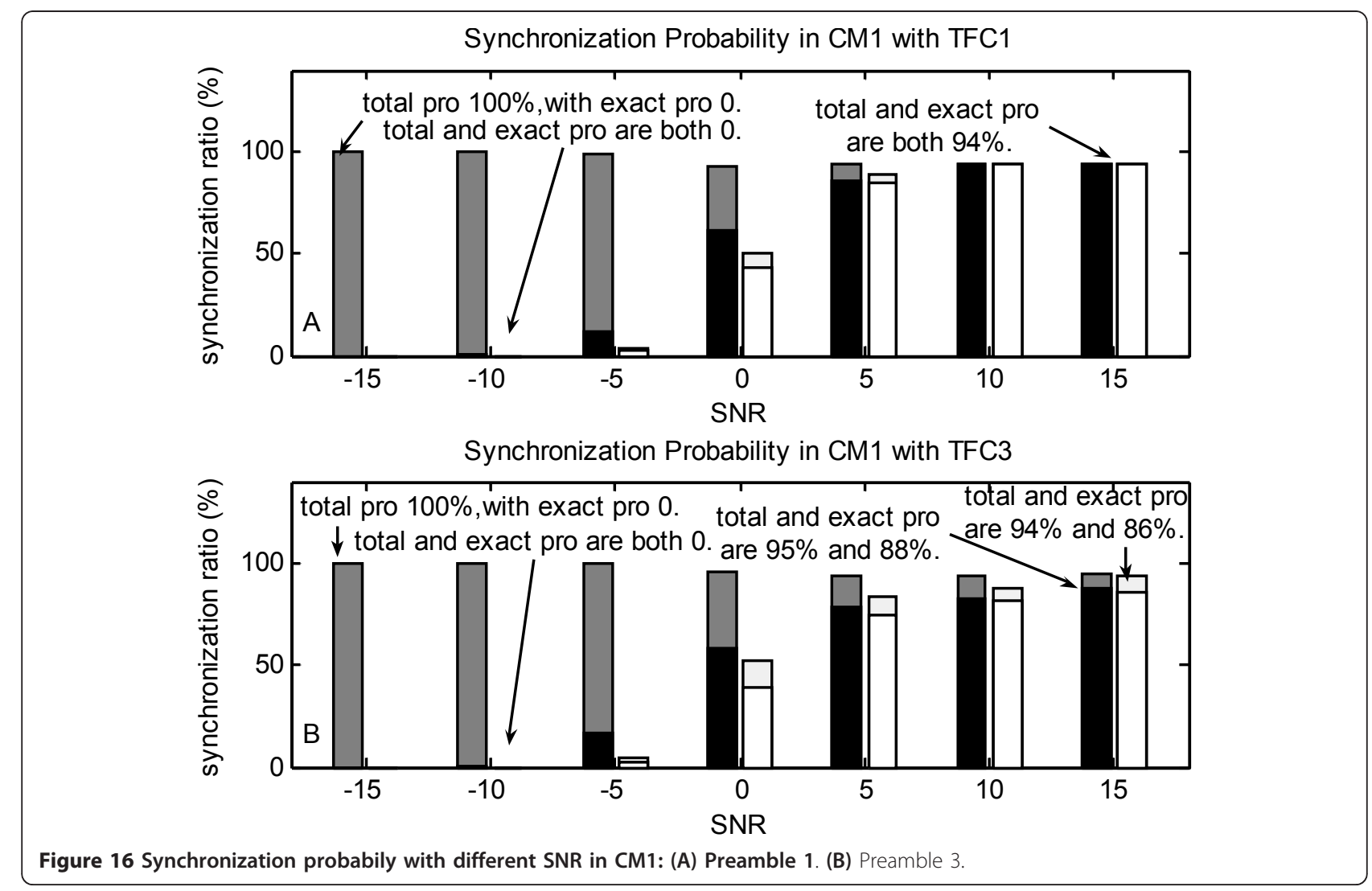




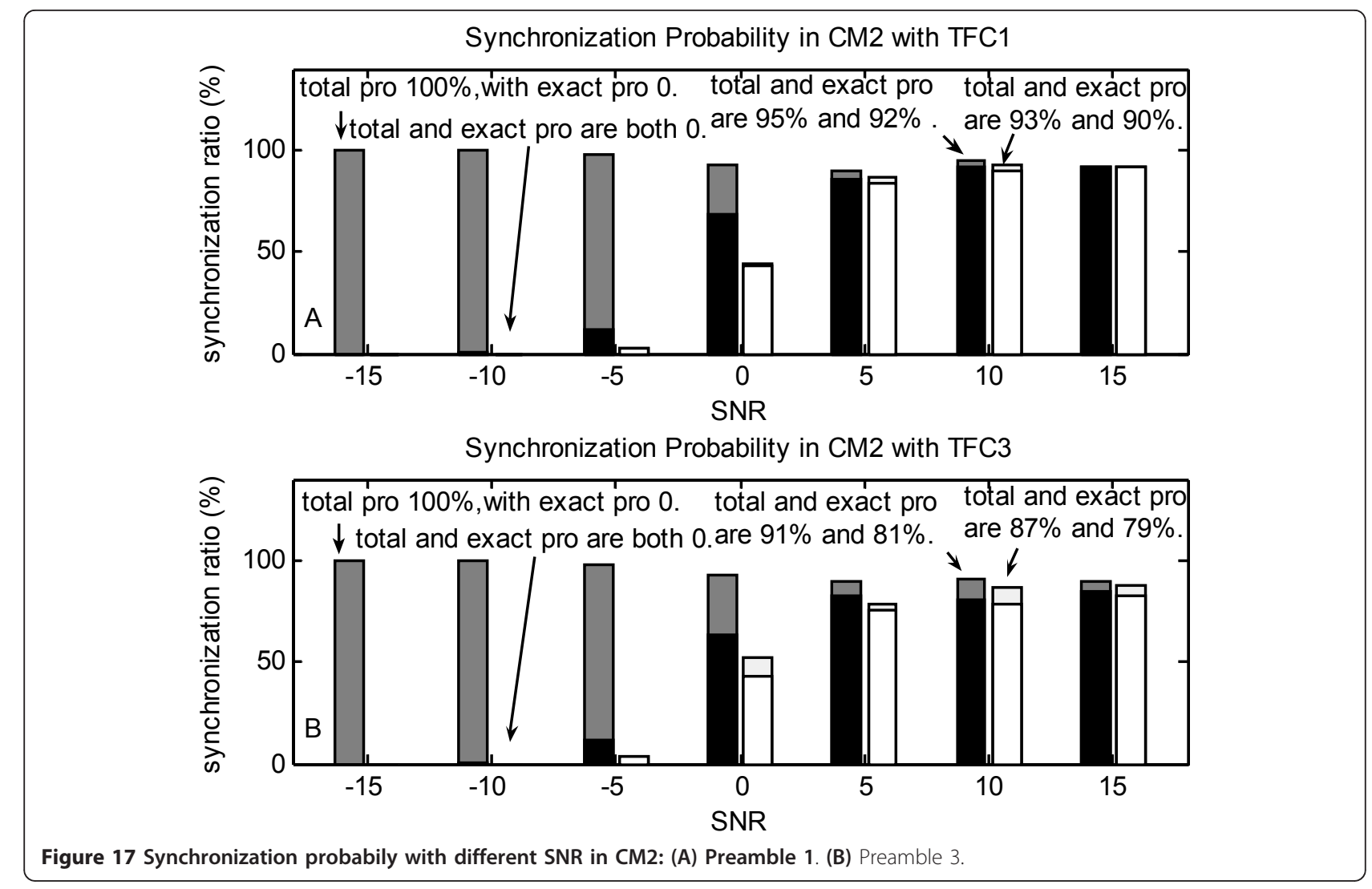

locates in ZP would not introduce ICI and ISI, which is also meaningful for promoting system capability. Thus, the proposed estimator suits for different SNR environments more with a constant threshold.

\subsection{Complexity}

Comparing to thresholds-based scheme, the proposed scheme has a maximum operation of $M$ more XOR operation and $(2 M-1)$ adding operation. Cross-correlation operations are only implemented in the first step of timing, which are also need in other thresholds based estimations. The second and third steps introduce $M$ more XOR operations, $(M-1)$ adding operations, and $M$ adding operations, respectively. The third step is not needed sometimes, so $M$ adding operations are not always needed. In situations of appropriate thresholds and/or not very low SNR, $M$ is only a small number (e. g., when threshold $=35, \mathrm{SNR}=0 \mathrm{~dB}, \mathrm{CM} 1 \mathrm{TFC} 1, M=$ 23.06 in average; when threshold $=45$, CM2 TFC3, $M=$ 10.48 in average). Although we have defined two steps, which seems to be of high complexity, but in fact we only introduce 10 to 20 times XOR and adding operations. Meanwhile, performances improvement (esp. synchronization probabilities in low SNR) achieved by our approach is really valuable, which are demonstrated in
Figures 16 and 17 . We could get $100 \%$ improvement over literature at most. Therefore, our approach introduces a not high complexity in implementation, but achieves a most $100 \%$ total synchronization probabilities improvement.

\section{Conclusion}

We have presented an enhanced timing synchronization estimator based on correlation difference timing scheme by comparing the preamble polarities and guaranteeing timing result uniqueness for MB-OFDM-based UWB systems. We develop our estimator to get a unique timing synchronization sample when threshold is set in a reasonable range. The range requirement is not critical. The proposed estimator reduces restriction of threshold to some extent, and makes it possible to estimate symbol timing sample index with a much smaller MSE. What's more, total and exact timing synchronization probabilities as much as 100 and $96 \%$ are achieved on basis of unique timing result. Meanwhile, the proposed estimator is appropriate for both preamble patterns 1 (or 2) and 3 (or 4), which is of significant value for analyzing MB-OFDM-based UWB systems. Our proposed approach can be applied to other preamble-based systems and threshold-based schemes. 


\section{Abbreviations}

CBTS: correlation-based symbol timing synchronization; CM: channel model: ECMA-368/369: European Computer Manufacturers Association-368/369; FCC: Federal Communications Commission; FS: frame synchronization; ICl: inter carrier interferences; IFFT/FFT: inverse fast Fourier transform/fast Fourier transform; ISI: inter symbol interferences; MB-OFDM: multi-band orthogonal frequency division multiplexing; MSE: mean square error; PS: packet synchronization; SNR: signal noise ratio; TFC: time frequency code; UWB: ultra-wide band; ZP: zero padded.

\section{Acknowledgements}

This study was supported by the National Natural Science Foundation of China (No. 61071073) and the Doctoral Fund of Ministry of Education of China (No. 20090061110043)

\section{Competing interests}

The authors declare that they have no competing interests.

Received: 12 October 2011 Accepted: 14 May 2012

Published: 14 May 2012

\section{References}

1. Federal Communications Commission Technical, Revision of part 15 of the commission's rules regarding Ultra-Wideband transmission systems First report and order, FCC 02-48, Washington DC, USA, (April 2002)

2. MZ Win, RA Scholtz, Impulse radio: how it works. IEEE Commun Lett. 2(2), 36-38 (1988)

3. MZ Win, RA Scholtz, Ultra-wide bandwidth time-hopping spread-spectrum impulse radio for wireless multiple-access communications. IEEE Trans Commun. 48(4), 679-691 (2000)

4. A Batra, J Balakrishnan, A Dabak, R Gharpurey, J Lin, P Fontaine, J-M Ho, S Lee, M Frechette, S March, $\mathrm{H}$ Yamaguchi, Multi-band OFDM physical layer proposal for IEEE 802.15 task group 3a, IEEE P802.15-03/268r3, Orlando, FL, USA, (March 2004)

5. E Cano, A Rabbachin, D Fuehrer, J Fortuny, On the evaluation of MB-OFDM UWB interference effects on a WiMAX receiver. EURASIP J Wirel Commun Netw. 2010, Article ID 414927, 14 (2010). doi:10.1155/2010/414927

6. IEEE P802.15 Wireless Personal Area Networks (WPANs) Group 3a, Multiband OFDM physical layer proposal for IEEE 802.15 task group 3a (March 2004)

7. Standard ECMA-368. High Rate Ultra Wideband Phy and Mac Standard, 1 st edn. (December 2005)

8. H Steendam, M Moeneclaey, Synchronization sensitivity of multi-carrier systems, in Eur Trans Commun, ETT special issue on multi-carrier spread spectrum), 52(5) 834-844 (2004)

9. $\mathrm{PH}$ Moose, A technique for orthogonal frequency division multiplexing frequency offset correction. IEEE Trans Commun. 42(10), 2908-2914 (1994)

10. M Morelli, U Mengali, An improved frequency offset estimator for OFDM applications. IEEE Commun Lett. 3(3), 75-77 (1999)

11. Y Li, T Jacobs, H Minn, Frequency offset estimation for MB-OFDM-based UWB systems, in Proceeding of IEEE International conference on Communications, Istanbul, 4729-4734 (June 2006)

12. Y Li, M Hlaing, T Jacobs, M Win, Frequency offset estimation for MB-OFDMbased UWB systems. IEEE Trans Commun. 56(6), 968-979 (2008)

13. H Minn, P Tarasak, VK Bhargava, OFDM frequency offset estimation based on BLUE principle, in Proceeding of IEEE 56th Vehicular Technology Conference, Vancouver, Canada, Fall. 2, 1230-1234 (2002)

14. H Minn, P Tarasak, VK Bhargava, Some issues of complexity and training symbol design for OFDM frequency offset estimation methods based on BLUE principle, in Proceeding of IEEE 57th Vehicular Technology Conference, Jcju, Korea, Spring. 2, 1288-1292 (2003)

15. H Minn, P Tarasak, Improved maximum likelihood frequency offset estimation based on likelihood metric design. IEEE Trans Signal Process. 54(6), 2076-2086 (2006)

16. $\mathrm{H}$ Yang, KS Jeong, JH Yi, YH You, Integer frequency offset estimator by frequency domain spreading for UWB multiband-OFDM. IEICE Trans Fund Electron Commun Comput Sci. E93A(3), 648-650 (2010)

17. TM Schmidl, DC Cox, Robust frequency and timing synchronization for OFDM. IEEE Trans Commun. 45(12), 1613-1621 (1997)

18. H Minn, M Zeng, VK Bhargava, On timing offset estimation for OFDM systems. IEEE Commun Lett. 4(7), 242-244 (2000)
19. B Park, H Cheon, C Kang, D Hong, A novel timing estimation method for OFDM systems. IEEE Commun Lett. 7(5), 239-241 (2003)

20. SD Choi, JM Choi, JH Lee, An initial timing offset estimation method for OFDM systems in rayleigh fading channel, in Proceeding of IEEE 64th Vehicular Technology Conference, Montreal, Canada, Fall, 1-5 (2006)

21. D Sen, S Chakrabarti, RVR Kumar, Some interesting results on compatible BER analysis issues related to multi-band timing and frequency synchronizers applicable for MB-OFDM based UWB communications. Digital Signal Process. 21(2), 332-340 (2011)

22. D Sen, S Chakrabarti, RVR Kumar, An adaptive timing synchronization scheme for multi-band orthogonal frequency division multiplexing based ultra-wideband communication systems. Wirel Personal Commun. 53(2), 281-298 (2010)

23. AM Karim, M Othman, E Zahedi, Packet synchronization structure with peak detection algorithm for MB-OFDM UWB, in Proceeding of IEEE International Conference on Semiconductor Electronics, Kuala Lumpur, 388-391 (October 29-December 1 2006)

24. CW Yak, Z Lei, TT Tjhung, Maximum likelihood frequency offset estimation and Cramer Rao Bound for Ultra-Wideband (UWB) multi-band OFDM systems, in Proceeding of IEEE 63th Vehicular Technology Conference, Melbourne, Australia, Spring, 2373-2377 (2006)

25. CW Yak, Z Lei, S Chattong, TT Tjhung, Timing synchronization for ultrawideband (UWB) multi-band OFDM systems, in Proceeding of IEEE 62th Vehicular Technology Conference, Dallas, USA, Fall, 1599-1603 (2005)

26. D Sen, S Chakrabarti, KRV Raja, A new timing estimation and compensation scheme for ultra-wideband communications, in Proceeding of IEEE 5th IFIP International conference on Wireless and Optical Communicaiton Networks, WOCN'08, Surabaya, East Java Indonesia, 1-5 (5-7 May 2008)

27. ZZ Ye, CJ Duan, PV Orlik, JY Zhang, AA Abouzeid, A synchronization design for UWB-based wireless multimedia systems. IEEE Trans Broadcast. 56(2), 211-225 (2010)

28. SH Yoon, JW Chong, Packet detection and symbol timing synchronization algorithm for multi-band OFDM UWB. IEICE Trans Commun. E89B(4), 1433-1435 (2006)

29. AF Molisch, JR Foerster, M Pendergrass, Channel models for ultrawideband personal area networks. IEEE Wirel Commun Mag. 10(6), 14-21 (2003)

doi:10.1186/1687-1499-2012-169

Cite this article as: Wang et al: A polarity comparison timing synchronization estimation for MB-OFDM-based UWB systems. EURASIP Journal on Wireless Communications and Networking 2012 2012:169.

\section{Submit your manuscript to a SpringerOpen ${ }^{\mathcal{O}}$ journal and benefit from:}

- Convenient online submission

- Rigorous peer review

- Immediate publication on acceptance

- Open access: articles freely available online

- High visibility within the field

- Retaining the copyright to your article

Submit your next manuscript at $\gg$ springeropen.com 